\title{
Space-filling branes of gravitational ancestry
}

\author{
Claudio Bunster ${ }^{*}$ and Alfredo Pérez \\ Centro de Estudios Cientificos (CECs), Avenida Arturo Prat 514, Valdivia, Chile
}

\begin{abstract}
We introduce a new kind of space-filling brane, which we term "G-brane" because its action is a descendant of the gravitational action. The G-brane may be thought of as the remanent of the gravitational field when the propagating gravitons are removed. The G-brane is different from the Dirac or Nambu space-filling branes. Its properties in any spacetime dimension $D$ are exhibited. When the spacetime dimension $D$ is greater than or equal to three, the G-brane does not possess propagating degrees of freedom, just as the Dirac or Nambu branes. For $D=3$ the G-brane yields a reformulation of gravitation theory in which the Hamiltonian constraints can be solved explicitly, while keeping the spacetime structure manifest. For $D=2$ the G-brane provides a realization of the conformal algebra, i.e. a conformal field theory, in terms of two scalar fields and their conjugates, which possesses a classical central charge. In the G-brane reformulation of $(2+1)$ gravity, the boundary degrees of freedom of the gravitational field in asymptotically anti-de Sitter space appear as "matter" coupled to the $(1+1)$ G-brane on the boundary.
\end{abstract}

PACS numbers: 04.20.Cv, 04.20.Fy, 11.25.-w

\section{INTRODUCTION}

The existence itself of the black hole, and the ensuing mystery of the origin of the black hole entropy, show that there is significant gravitational physics in vacuum which is not due to propagating gravitons. This verity is strikingly evidenced by the existence of a black hole in $2+1$ spacetime dimensions [1, 2]. One would like therefore to have a theory which captures what is left of gravitation after the propagating gravitons are removed. To be of general applicability such a descendant of general relativity should retain the general covariance of its ancestor.

We propose herein that the "gravitational field without propagating gravitons" may be described by a new kind of space-filling brane, which we term "G-brane," whose action is a direct descendant of the action for the gravitational field. The G-brane is different from the Dirac or Nambu space-filling branes.

We develop the general G-brane formalism for an arbitrary spacetime dimension $D$ and focus in detail on the lower dimensions $D=3$ and $D=2$. For $D=3$ the G-brane yields a reformulation of gravitation theory in which the Hamiltonian constraints can be solved explicitly, while keeping the spacetime structure manifest. For $D=2$, the G-brane provides a realization of the conformal algebra, i.e. a conformal field theory, in terms of two scalar fields and their conjugates, which possesses a classical central charge. In the G-brane reformulation of $(2+1)$ gravity, the boundary degrees of freedom of the gravitational field in asymptotically anti-de Sitter space appear as "matter" coupled to the $(1+1)$ G-brane on the boundary.

Many years ago [3] an attempt was made to reformulate Einstein's theory of gravitation in $D$ dimensions,

*Electronic address: bunster@cecs.cl

${ }^{\dagger}$ Electronic address: aperez@cecs.cl as the theory of a non-space-filling brane, embedded in a higher dimensional fixed (flat) spacetime background. However, obstacles that could not be satisfactorily circumvented were met (see also [4] and, for later developments, [5, 6]). Those difficulties are not present for the space-filling brane.

The plan of the paper is the following. Section II reviews and puts in context the concepts of Dirac and Nambu space-filling branes. Section III introduces the G-brane, and discusses its general properties for $D \geq 3$, while Sec. IV discusses the special case of the G-brane for $D=2$. Section $\mathrm{V}$ contains the reformulation of gravitation in three spacetime dimensions as the theory of a G-brane. Finally Sec. VI is devoted to conclusions and possible further developments along this line of inquiry.

\section{DIRAC AND NAMBU SPACE-FILLING BRANES}

\section{A. Surface deformations and surface variables}

Space-filling branes were first introduced by Dirac in his formulation of field dynamics on an arbitrary spacelike surface, within a fixed background spacetime that he mostly took to be flat [7]. He introduced surface variables $y^{\lambda}(x, t)$, which describe the embedding of a parametrized spacelike surface with respect to a fixed system of coordinates $y^{\lambda}$ on the background, and their conjugate momenta $w_{\lambda}$. Thus, in terms of Poisson brackets,

$$
\left[y^{\lambda}(x), w_{\rho}\left(x^{\prime}\right)\right]=\delta_{\rho}^{\lambda} \delta\left(x, x^{\prime}\right) .
$$

The Hamiltonian for a field theory in which the $t=$ constant surface is a generic spacelike surface was then written as

$$
H=\int d x N^{\lambda} h_{\lambda},
$$


where the generators $h_{\lambda}$ have the form

$$
h_{\lambda}=w_{\lambda}-T_{\lambda \rho} n^{\rho} .
$$

The action reads

$$
I=\int d x d t\left(w_{\lambda} \dot{y}^{\lambda}+\pi \dot{\varphi}-N^{\lambda} h_{\lambda}\right) .
$$

Here $n^{\rho}$ is the unit normal to the surface,

$$
\begin{aligned}
\gamma_{\lambda \rho} n^{\lambda} n^{\rho} & =-1 \\
n_{\lambda} y^{\lambda}{ }_{, i} & =0 .
\end{aligned}
$$

The components $n^{\rho}$ are functions of the $y$ 's and their first spatial derivatives. If $n^{\rho}$ solves (2) and (3), so does $-n^{\rho}$ corresponding to time reversal. We have denoted by $\gamma_{\lambda \rho}$ the metric in the external coordinate system in order to reserve the letter $g$ for the spatial metric $g_{i j}$ induced on the parametrized surface:

$$
g_{i j}[y](x)=\gamma_{\lambda \rho}(y(x)) y_{, i}^{\lambda} y_{, j}^{\rho} .
$$

The $T_{\lambda \rho}$ are the components of the symmetric energymomentum tensor of the matter fields at hand, with appropriate density weight. The projections $T_{\perp \perp}=$ $T_{\lambda \rho} n^{\lambda} n^{\rho}$ and $T_{\perp i}=T_{\lambda \rho} n^{\lambda} y^{\rho}{ }_{i}$, which are the energymomentum densities, depend on the matter canonical variables $\pi, \varphi$, and on the $y^{\lambda}$, but they do not depend on the $w_{\lambda}$.

The $N^{\lambda}$ are Lagrange multipliers for the constraints,

$$
h_{\lambda} \approx 0,
$$

in Dirac's "weakly vanishing" terminology and notation [8].

The generators $h_{\lambda}$ may be decomposed into a component $h_{\perp}$ normal to the surface and $h_{i}$ tangential to it, that is:

$$
\begin{aligned}
h_{\perp} & =\left(w_{\lambda}-T_{\lambda \rho} n^{\rho}\right) n^{\lambda}=w_{\perp}-T_{\perp \perp}, \\
h_{i} & =\left(w_{\lambda}-T_{\lambda \rho} n^{\rho}\right) y^{\lambda}{ }_{, i}=w_{i}-T_{\perp i} .
\end{aligned}
$$

The corresponding multipliers are then the "lapse," and "shift" functions, which are related to the $N^{\lambda}$ through

$$
N^{\lambda}=N^{\perp} n^{\lambda}+N^{i} y^{\lambda}, i
$$

The equations of motion of the $y^{\lambda}$ are

$$
\dot{y}^{\lambda}=N^{\perp} n^{\lambda}+N^{i} y_{, i}^{\lambda},
$$

which, since $N^{\perp}$ and $N^{i}$ are arbitrary functions of time, express the fact that any deformation of the brane is permissible. The equations of motion for the matter fields describe their response to a surface deformation.

\section{B. Surface deformation algebra}

The perpendicular and tangential projections are useful because $h_{\perp}$ generates deformations of the surface which are of dynamical importance, whereas $h_{\perp i}$ merely reparametrizes the spacelike surface without really moving away from it. Note that this decomposition makes essential use of the spacetime metric in order to give an unambiguous meaning, through the normal, to the notion of "moving away from the surface." Thus the notion of surface deformation expressed in terms of the projected generators is different from, and has much more structure than, the notion of a spacetime diffeomorphism. The surface deformation generators must obey integrability conditions that guarantee that the evolution is "path independent": if one starts from a given initial spacelike surface and ends on a given final spacelike surface, the result should be independent of the intermediate sequence of surfaces employed to achieve the total deformation as a sequence of infinitesimal ones [9, 10]. These integrability conditions read, in terms of the unprojected generators,

$$
\left[h_{\lambda}(x), h_{\rho}\left(x^{\prime}\right)\right]=0,
$$

or in terms of the projected ones,

$$
\begin{aligned}
{\left[h_{\perp}(x), h_{\perp}\left(x^{\prime}\right)\right] } & =g^{i j}\left(h_{i}(x)+h_{i}\left(x^{\prime}\right)\right) \delta_{, j}\left(x, x^{\prime}\right), \\
{\left[h_{i}(x), h_{\perp}\left(x^{\prime}\right)\right] } & =h_{\perp}(x) \delta_{, i}\left(x, x^{\prime}\right), \\
{\left[h_{i}(x), h_{j}\left(x^{\prime}\right)\right] } & =h_{i}\left(x^{\prime}\right) \delta_{, j}\left(x, x^{\prime}\right)+h_{i}(x) \delta_{, i}\left(x, x^{\prime}\right) .
\end{aligned}
$$

The last two equations, which involve $h_{i}$ on the left-hand side are of purely kinematical nature since they just capture how $h_{\perp}$ and $h_{i}$ behave under changes in the spatial coordinates. The truly restrictive equation is the first one (8) which imposes severe conditions on the components of the energy-momentum densities. Its quantum mechanical counterpart was referred to by Schwinger in the concluding sentence of [11] as "what may well be considered the most fundamental equation of relativistic quantum field theory."

\section{Dirac brane}

If one drops the matter fields in the above equations, one has

$$
T_{\lambda \rho}=0,
$$

and the generators read

$$
h_{\lambda}=w_{\lambda},
$$

or in their projected version,

$$
\begin{aligned}
h_{\perp} & =w_{\perp}, \\
h_{i} & =w_{i} .
\end{aligned}
$$


The generators $w_{\perp}$ and $w_{i}$ obey the algebra (8)-(10) for any background, and they generate a consistent evolution for a space-filling brane that we will call a Dirac brane. Equations (1), (5) and (6) may then be said to describe the coupling of a Dirac brane to matter.

For the Dirac brane without matter fields, the action in Hamiltonian form reads

$$
I=\int d x d t\left(w_{\lambda} \dot{y}^{\lambda}-N^{\lambda} w_{\lambda}\right)
$$

where $g$ is the determinant of the spatial metric $g_{i j}$. If one passes to the Lagrangian form of the action by eliminating $w_{\lambda}$ from the constraint, one gets

$$
I=0 \text {. }
$$

\section{Nambu brane}

A different kind of space-filling brane, which we shall call Nambu brane for reasons that will become evident in a moment, is obtained by demanding that there should be a constant energy density $\mathcal{N}$ through space, that is, one sets,

$$
T_{\lambda \rho}=\mathcal{N} g^{1 / 2} \gamma_{\lambda \rho}
$$

The generators now read

$$
h_{\lambda}=w_{\lambda}-\mathcal{N} g^{1 / 2} n_{\lambda}
$$

or

$$
\begin{aligned}
h_{\perp} & =w_{\perp}+\mathcal{N} g^{1 / 2}, \\
h_{i} & =w_{i},
\end{aligned}
$$

and they obey the integrability conditions (7)-(10), for any background.

For the Nambu brane the action in Hamiltonian form may be written as

$$
I=\int d x d t\left(w_{\lambda} \dot{y}^{\lambda}-N^{\lambda}\left(w_{\lambda}-\mathcal{N} g^{1 / 2} n_{\lambda}\right)\right),
$$

whereas the Lagrangian form now reads

$$
\begin{aligned}
I & =\mathcal{N} \int d x d t\left(g^{1 / 2} n_{\lambda} \dot{y}^{\lambda}\right), \\
& =-\mathcal{N} \int d x d t\left(-g_{\text {spacetime }}\right)^{1 / 2}
\end{aligned}
$$

which is indeed the action for a (space-filling) Nambu brane. If one has a matter energy-momentum tensor, obeying the appropriate Poisson bracket rules which make the Dirac brane generators (5) and (6) to close according to (8) - (10), then its addition to the Nambu brane generators will preserve the closure.

The Lagrangian action for the Nambu brane (12) is invariant under spacetime reparametrizations,

$$
y^{\lambda}(x, t) \rightarrow y^{\lambda}(x, t)+\delta y^{\lambda}(x, t) .
$$

More precisely, if the integral in (12) is extended over a spacetime region $M$ with boundary $\partial M$ one has

$$
\delta I=\int_{\partial M} d x\left(\mathcal{N} g^{1 / 2} n_{\lambda} \delta y^{\lambda}\right)
$$

which is where (11) may be thought to come from in the first place.

We now turn our attention to the main point of the paper, a new type of brane.

\section{G-BRANE FOR $D \geq 3$}

\section{A. Action}

A different type of space-filling brane is obtained by replacing the spacetime volume (12) by a different invariant, the action for the gravitational field evaluated on a given background. Hence the name G-brane.

The Lagrangian action for the gravitational field is

$$
I_{\text {grav }}=\int d t d x N^{\perp} \mathcal{L}
$$

with

$$
\mathcal{L}=\frac{1}{2 \kappa}\left(G^{i j k l} K_{i j} K_{k l}+g^{1 / 2}(R-2 \Lambda)\right),
$$

and

$$
G^{i j k l}=\frac{1}{2} g^{1 / 2}\left(g^{i k} g^{j l}+g^{i l} g^{j k}-2 g^{i j} g^{k l}\right) .
$$

Here $K_{i j}$ denotes the extrinsic curvature of the $t=$ constant surface, which is the "invariant velocity of $g_{i j}$ "

$$
\dot{g}_{i j}=-2 N^{\perp} K_{i j}+N_{i / j}+N_{j / i},
$$

and $\kappa$ is related to Newton's gravitational constant by $\kappa=8 \pi G$.

The action (15) differs with the Hilbert action by adding to it a divergence which removes the second time derivatives of $g_{i j}$ and also the second and first derivative of $g_{0 \mu}$.

Under a spacetime reparametrization the analog of (13) is

$$
\delta I=-\int_{\partial M} d x\left[\mathcal{L} n_{\lambda} \delta y^{\lambda}\right]
$$

which yields the G-brane generators,

$$
h_{\lambda}=w_{\lambda}+\mathcal{L} n_{\lambda}
$$

whose projected version is

$$
\begin{aligned}
h_{\perp} & =w_{\perp}-\mathcal{L}, \\
h_{i} & =w_{i} .
\end{aligned}
$$


Here the extrinsic curvature is understood to be expressed in terms of the surface variables $y^{\lambda}$, by the counterpart,

$$
K_{i j}=n_{\lambda} D_{i} y_{, j}^{\lambda}
$$

of (44), where $D_{j}=y^{\lambda}{ }_{j} D_{\lambda}$ is the covariant derivative in the external space projected on the $t=$ constant surface.

For the G-brane the action in Hamiltonian form reads

$$
I=\int d x d t\left[w_{\lambda} \dot{y}^{\lambda}-N^{\lambda}\left(w_{\lambda}+\mathcal{L} n_{\lambda}\right)\right],
$$

and the Lagrangian form is

$$
I=-\int d x d t\left[\mathcal{L} n_{\lambda} \dot{y}^{\lambda}\right] .
$$

Equations (18) and (19) were obtained many years ago [3] in an attempt to reformulate Einstein's gravity in $D$ dimensions as the theory of a non-space-filling brane embedded in a higher dimensional (flat) spacetime background. In that case one cannot express the extrinsic curvature in terms of the surface variables through (20) and the need for imposing additional constraints arises. It was proposed in [3] that these additional constraints might be the standard gravitational initial valued constraints, but the analysis was not carried to completion (see also [4] and, for later developments, [5, 6] ).

\section{B. Integrability and closure}

The generators $h_{\lambda}$ given by (17) close according to (7), which is equivalent to (8)-(10) because on account of (16) one has

$$
\mathcal{L} n_{\lambda}=-\frac{\delta I}{\delta y^{\lambda}(x)} .
$$

Here $I$ is the gravitational action evaluated on the given background, for a spacetime region bounded by an initial and a final spacelike surface, regarded as a functional of the location of the final surface $y^{\lambda}(x)$.

It is essential for the action integral $I$ to be well defined as a functional of the final surface, that it be invariant under reparametrizations of the spacetime region in between the two surfaces. This invariance guarantees that the value obtained for the action is independent of the particular foliation by $t=$ constant surfaces employed in carrying out the integration.

Note that in order for the resulting G-brane action to depend only on the $y^{\lambda}$ and his first time derivatives, the associated gravitational background action should depend only on the $g_{i j}$ and his first time derivatives.

For $D=3$ and $D=4$ spacetime dimensions the only possibility for the action $I$ appearing in (22) is (15), which may be understood as the "dimensional continuation" of the Euler classes of the lower even dimensions
$D=0$, corresponding to the cosmological term (Nambu brane) and $D=2$ (gravitational action without cosmological term).

For higher spacetime dimensions $D$ there are more possible background actions, the so-called Lovelock Lagrangians [12], which may be understood as the dimensional continuation of all the Euler classes of the even dimensions below $D$. The G-brane action arising from the generic Lovelock Lagrangian is then a sum of terms each of which involves powers of the intrinsic Riemann tensor of the surface and its extrinsic curvature [13].

\section{Time reversal}

The ancestor action (14) is invariant under time reversal $t \rightarrow-t$ because, although $K_{i j} \rightarrow-K_{i j}$ the Lagrangian (15) is quadratic in $K_{i j}\left(g_{i j}\right.$ and $N^{\perp}$ are invariant). However the generator $h_{\perp}$ given by (18) is not invariant because under time reversal $n^{\lambda} \rightarrow-n^{\lambda}$ and therefore $w_{\perp}$ changes sign while $\mathcal{L}$ does not. The situation is exactly the same as the one for a relativistic particle where

$$
p_{\lambda} p^{\lambda}+m^{2}=0
$$

is equivalent to

$$
p^{0} \mp \sqrt{\vec{p}^{2}+m^{2}}=0 .
$$

Here as well the G-brane generators have two branches, which are automatically included in the description, because Eq. (17) does not specify which of the two solutions of the equations (2) and (3) differing by a sign is chosen.

One may replace the generator (18) by the combination

$$
h=w_{\lambda} w^{\lambda}+\mathcal{L}^{2},
$$

an expression whose appearance is familiar from the bosonic string. The quadratic generator (23) incorporates both, $h_{\perp}$ and its time reversed version. There are cases of interest for which quadratic the form (23) is considerably simpler than its linear counterpart, notably the Born-Infeld electrodynamics as reformulated by Dirac [14]. However from a geometrical point of view 23] has the drawback that the bracket of two such generators at different spatial points close in a way, which is not universal (the structure coefficients of the constraint algebra depend on the matter fields).

\section{Background energy}

Formally the G-brane, and the Nambu brane, are related to the Dirac brane by a point canonical transformation, whose generating functional is precisely $I$ appearing in Eq. (22).

However, already for the case of the Nambu brane, one has reason to believe that if the framework is enlarged a 
bit, the vacuum energy represented by the cosmological term cannot simply be subtracted. One knows, for example, that if one introduces $(D-2)$ branes endowed with a $U(1)$ charge, the reservoir of vacuum energy can be tapped [15, 16], and it even can be used to form black holes [17].

Although at present no similar mechanism is known for the other terms in $\mathcal{L}$, they also represent a sort of "gravitational energy of the background," which prudence would advise to retain.

Of course the value of the terms in $g^{-1 / 2} \mathcal{L}$ other than the cosmological term $-\kappa^{-1} \Lambda$ depend on the surface $y^{\lambda}(x)$. In flat spacetime one may choose a surface of constant Minkowskian time and both the intrinsic and extrinsic curvatures will then vanish. However, if one is on a curved background, a surface where $\mathcal{L}$ vanishes will in general not exist. For example, in an expanding section of the de Sitter universe, the intrinsic curvature term vanishes, but the extrinsic one does not. It, rather, doubles the cosmological term contribution.

Conceptually, the issue of the background energy is not different from the discussion of the dependence of the inertia of a body upon its energy content [18]. In that case, if one stays within the strict context of the dynamics of a relativistic particle that does not decay, whether one favors the more elegant expression,

$$
E=\frac{m_{0}}{\sqrt{1-v^{2} / c^{2}}}
$$

over

$$
E=\frac{m_{0}}{\sqrt{1-v^{2} / c^{2}}}-m_{0} c^{2}
$$

which reduces to the nonrelativistic expression when $c \rightarrow$ $\infty$, is a matter of taste, with no physical implication.

But if the particle can decay, so that the rest mass reservoir can be tapped, then the first choice becomes mandatory.

Before leaving this point we remark that $\mathcal{L} n_{\lambda}$ cannot be interpreted as the components $T_{\perp \lambda}$ of a tensor. In particular they bear no relation to the conserved energymomentum tensor $T^{\lambda \rho}$ of the G-brane, obtained by varying the action (21) with respect to the background metric $\gamma_{\lambda \rho}$, which is given by

$$
T_{\lambda \rho}=\kappa^{-1} G_{\lambda \rho}
$$

where $G_{\lambda \rho}$ is the Einstein tensor of the background.

Therefore if one attempts to turn the G-brane into a source for the gravitational field, the Einstein's equations are identically satisfied. Hence the coupling of the G-brane to its ancestor is not achieved. This is quite in line with the interpretation of the G-brane as gravitation without gravitons, because when one turns on the full dynamical gravitational field, the G-brane should be automatically included rather than being an additional "matter" source.

\section{G-BRANE FOR $D=2$}

\section{A. Action}

As we saw in the previous section, for spacetime dimensions $D \geq 3$ the G-brane action is a descendant of the action for the gravitational field. For $D=2$ there is an analog of the Einstein theory of gravitation, which has similarities with its higher dimensional counterpart but also possesses key differences with it. The similarities and differences of their gravitational ancestors are inherited by their descendants, the G-branes.

For two spacetime dimensions the analog of the Einstein equation,

$$
R_{\mu \nu}-\frac{1}{2} g_{\mu \nu} R+\Lambda g_{\mu \nu}=0
$$

is,

$$
R-\Lambda=0
$$

In both cases the sign of $R$ is the same as the sign of $\Lambda$.

Equation (24) may be derived [19, 20] from an action which is built out of the spacetime metric $g_{\mu \nu}$. That action shares with its higher dimensional counterparts the properties of depending only on the first derivative of the spatial metric $g_{11}$ and of containing no time derivatives of the $g_{0 \mu}$; but it has the important difference of not being invariant under spacetime reparametrizations.

However the lack of invariance is of such a especial nature that it makes it still possible to define from it a consistent set of surface deformation generators. The price paid is the appearance of a central charge in the surface deformation algebra, which has the consequence that the generators cannot be demanded to vanish. Due to the absence of initial value constraints, $D=2$ gravity has one independent degree of freedom per point, rather than "minus one" as extrapolated count from higher dimensions would naively indicate. A similar situation will arise for its descendant, the $(1+1)$ G-brane.

In [19] the metric of the two-dimensional spacetime, referred to the "internal" coordinates $t, x$, was written as

$$
g_{\mu \nu}=e^{\varphi}\left[\begin{array}{cc}
-\left(\eta^{\perp}\right)^{2}+\left(\eta^{1}\right)^{2} & \eta^{1} \\
\eta^{1} & 1
\end{array}\right] .
$$

Here the rescaled lapse $\eta^{\perp}=e^{-\varphi / 2} N^{\perp}$ and the customary shift $\eta^{1}=N^{1}$, describe a generic deformation of a parametrized one-dimensional surface embedded in a two-dimensional spacetime in a conformally invariant manner, through the introduction of a Weyl invariant normal $\tilde{n}^{\lambda}$ which differs from the unit normal $n^{\lambda}$ by a scale factor. That is one writes

$$
\begin{aligned}
\dot{y}^{\lambda} & =\eta^{\perp} \tilde{n}^{\lambda}+\eta^{1} y^{\lambda}{ }_{, 1}, \\
\gamma_{\lambda \rho} \tilde{n}^{\lambda} \tilde{n}^{\rho} & =-g_{11} \equiv-g .
\end{aligned}
$$

Under a spacetime dependent rescaling of the metric $\gamma_{\lambda \rho}(y) \rightarrow e^{\sigma(y)} \gamma_{\lambda \rho}(y)$ the function $\varphi$ changes as $\varphi \rightarrow$ 
$\varphi+\sigma$, whereas $y^{\lambda}, \tilde{n}^{\lambda}, \eta^{\perp}, \eta^{1}$ remain invariant. The Weyl invariant normal $\tilde{n}^{\lambda}$ is related to the unit normal used before by

$$
\tilde{n}^{\lambda}=g^{1 / 2} n^{\lambda} .
$$

The Lagrangian density reads

$$
\mathcal{L}=\frac{1}{4 k}\left(2 e^{\varphi} K^{2}-\frac{1}{2} \varphi^{\prime 2}+2 \varphi^{\prime \prime}+\Lambda e^{\varphi}\right),
$$

where the extrinsic curvature $K$ is understood to be expressed in terms of the surface variables $y^{\lambda}$,

$$
K=g^{-3 / 2} \tilde{n}_{\lambda} D_{1} y^{\lambda}, 1 .
$$

Here $D_{1}=y^{\lambda}{ }_{1} D_{\lambda}$ is the covariant derivative in the external space projected on the $t=$ constant surface. The " $(1+1)$ gravitational constant $k$ " has the dimensions of an inverse action.

The action is given by

$$
I_{\text {grav }}=\int d t d x \eta^{\perp} \mathcal{L} .
$$

\section{B. Integrability and closure. Classical central charge}

If one reparametrizes by

$$
\delta y^{\lambda}=\xi^{\perp} \tilde{n}^{\lambda}+\xi^{1} y^{\lambda}, 1,
$$

the action for a spacetime region $M$ with boundary $\partial M$ changes by

$$
\delta I_{\text {grav }}=\int_{\partial M} \xi^{\perp} \mathcal{L}+\frac{1}{k} \int_{M}\left(\eta^{\perp} \xi^{1^{\prime \prime \prime}}-\xi^{\perp} \eta^{1^{\prime \prime \prime}}\right) .
$$

If the volume term on the right-hand side of the above equation were absent, the differential $\delta I_{\text {grav }}$ would be exact, that is the integral $I_{\text {grav }}$ would not depend on the particular foliation of $M$ used to calculate it. This is the case for the G-brane discussed in the preceding section and it implies that the generators,

$$
s_{\lambda}=w_{\lambda}+g^{-1} \mathcal{L} \tilde{n}_{\lambda},
$$

commute, i.e., that their Poisson bracket vanishes identically.

However when the differential $\delta I_{\text {grav }}$ is not exact, its second functional derivatives do not commute, and therefore the brackets of two $s_{\lambda}$ is not zero. The lack of commutativity of the $s_{\lambda}$ translates into a modification of the surface deformation algebra, which may be read directly from (29). One obtains for the projected form of the generators,

$$
\begin{aligned}
{\left[s_{\perp}(x), s_{\perp}\left(x^{\prime}\right)\right] } & =\left(s_{1}(x)+s_{1}\left(x^{\prime}\right)\right) \delta^{\prime}\left(x, x^{\prime}\right), \\
{\left[s_{1}(x), s_{\perp}\left(x^{\prime}\right)\right] } & =\left(s_{\perp}(x)+s_{\perp}\left(x^{\prime}\right)\right) \delta^{\prime}\left(x, x^{\prime}\right) \\
& -\frac{1}{k} \delta^{\prime \prime \prime}\left(x, x^{\prime}\right), \\
{\left[s_{1}(x), s_{1}\left(x^{\prime}\right)\right] } & =\left(s_{1}(x)+s_{1}\left(x^{\prime}\right)\right) \delta^{\prime}\left(x, x^{\prime}\right) .
\end{aligned}
$$

From these equations one may work "backwards" to obtain the Poisson bracket of the unprojected $s_{\lambda}$. This gives

$$
\begin{aligned}
{\left[s_{\lambda}(x), s_{\rho}\left(x^{\prime}\right)\right]=} & \frac{1}{k}\left(a_{\lambda}(x) b_{\rho}\left(x^{\prime}\right)+a_{\rho}\left(x^{\prime}\right) b_{\lambda}(x)\right) \times \\
& \times \delta^{\prime \prime \prime}\left(x, x^{\prime}\right),
\end{aligned}
$$

where $a_{\alpha}$ and $b_{\alpha}$, which are Weyl invariant, are given by

$$
a_{\alpha}=g^{-1} \gamma_{\alpha \beta} y_{, 1}^{\beta}, \quad b_{\alpha}=g^{-1} \gamma_{\alpha \beta} \tilde{n}^{\beta} .
$$

One sees that the $s_{\lambda}$ indeed do not commute.

Equations (30)-(32) for the Poisson brackets of the projected generators differ from the surface deformation algebra (8)-(10) on the following aspects:

(i) The coefficient of $\delta^{\prime}$ on the right side of (31) differs from that on (9), because on account of the normalization $h_{\perp}$ has now weight two instead of weight one as previously.

(ii) The metric $g^{i j}$ drops out from the right side of (30). This is a consequence of the conformally invariant normalization (27) for $\tilde{n}^{\alpha}$, and it only happens in one spatial dimension. The whole algebra is then a true algebra, in the sense that its structure constants are indeed constants rather than being field dependent.

(iii) A central charge appears. Since the central charge has vanishing Poisson brackets with everything the surface deformations are still integrable. As shown by Eq. (29) the central charge can be read directly from the lack of reparametrization invariance of the action. The fact that, because the charge is central, the second term on the right side of (29) is independent of the field $\varphi$, is responsible for the covariance of the equation of motion (24).

The surface deformation algebra in the form (30)-(32) is the Lie algebra of the conformal group, which consists of two copies of the Virasoro algebra,

$$
\left[L(x), L\left(x^{\prime}\right)\right]=\left(L(x)+L\left(x^{\prime}\right)\right) \delta^{\prime}\left(x, x^{\prime}\right)-\frac{1}{2 k} \delta^{\prime \prime \prime}\left(x, x^{\prime}\right),
$$

whose generators are

$$
L^{ \pm}(x)=(1 / 2)\left(s_{\perp}( \pm x) \pm s_{1}( \pm x)\right) .
$$

With the standard convention the Virasoro central charge $c$ appearing in (33) is given by

$$
c=\frac{12 \pi}{k} \text {. }
$$

Since, as a consequence of the presence of the central charge, the surface deformation generators cannot be required to vanish, the $s_{\lambda}$ are not to be regarded as generators of a gauge transformation but, rather, they generate a global symmetry transformation. The $(1+1)$ G-brane is then a novel conformal field theory with 2 degrees of freedom $y^{\lambda}$ per point, as it is highlighted in [21]. Moreover it will also prove useful in connection with asymptotic symmetries of $(2+1)$ gravity formulated in terms of the G-brane, which is the subject of the next section. 


\section{GRAVITATION IN THREE SPACETIME DIMENSIONS AS THE THEORY OF A G-BRANE}

The standard Einstein theory of gravitation in threedimensional spacetime is remarkable in the sense that it keeps the formal structure of its higher dimensional counterparts, but it has no field degrees of freedom associated to each point of two-dimensional space at a given time. In spite of this lack of "bulk degrees of freedom," when the cosmological constant is negative, $(2+1)$ gravity possesses black holes. It also has a peculiar structure at spatial infinity which is not present in higher dimensions, namely an infinite dimensional global symmetry.

However, in spite of its simplicity, the theory has so far only been solved explicitly in a reformulation in terms of a Chern-Simons connection. The Chern-Simons description has its own elegance and interest but, when one develops it, becomes disconnected from the original metric formulation and hence from spacetime.

The purpose of this section is to present a different reformulation of the theory, which is very close to, and actually suggested by, the metric formulation. Its spacetime significance is direct, even more direct than that in terms of the metric, and it stays manifest throughout. The reformulation also permits to solve the theory explicitly.

In three spacetime dimensions there is locally only one solution of Einstein's equations in vacuum, namely a spacetime of constant curvature, which up to identifications is anti-de Sitter space $(\Lambda<0)$, flat space $(\Lambda=0)$, or de Sitter space $(\Lambda>0)$. The absence of bulk degrees of freedom suggests that the theory should have a Gbrane formulation in terms of slices through a spacetime of constant curvature. We shall focus on the case $\Lambda<0$ because it has more structure: black holes and surface dynamics at spatial infinity.

\section{A. Surface variables as potentials that solve the constraints}

Our goal is thus to formulate the theory employing the potentials $y^{\lambda}$ and their conjugate momenta $w_{\lambda}$ as the fundamental variables, instead of the spatial metric $g_{i j}$ and its conjugate momentum $\pi^{i j}$. To that end we start with the "bulk" gravitational action in Hamiltonian form,

$$
I_{\text {grav }}=\int d t d x\left(\pi^{i j} \dot{g}_{i j}-N^{\perp} \mathcal{H}_{\perp}-N^{i} \mathcal{H}_{i}\right)
$$

where

$$
\begin{aligned}
\mathcal{H}_{\perp} & =2 \kappa G_{i j k l} \pi^{i j} \pi^{k l}-\frac{1}{2 \kappa} g^{1 / 2}(R-2 \Lambda), \\
\mathcal{H}_{i} & =-2 \pi_{i / j}^{j}
\end{aligned}
$$

with

$$
G^{i j k l} G_{k l r s}=\frac{1}{2}\left(\delta_{r}^{i} \delta_{s}^{j}+\delta_{s}^{i} \delta_{r}^{j}\right) .
$$

If one substitutes in (34) the relationship between the momentum and the velocity,

$$
\pi^{i j}=-\frac{1}{2 \kappa} G^{i j k l} K_{k l},
$$

one recovers the Lagrangian form (15).

The key observation now is that, if one takes $\gamma_{\lambda \rho}$ to be the anti-de Sitter metric, and substitutes expressions (4) and (20) in the generators $\mathcal{H}_{\perp}$ and $\mathcal{H}_{i}$ appearing in (34), then they vanish identically,

$$
\begin{aligned}
\mathcal{H}_{\perp} & \equiv 0, \\
\mathcal{H}_{i} & \equiv 0 .
\end{aligned}
$$

Conversely the most general solution of the constraint equations is obtained in this way, because they are precisely the equations of Gauss and Codazzi, which guarantee local embeddability. One may think of the GaussCodazzi equations implying the existence of the potentials $y^{\lambda}$ for $g_{i j}$ and $\pi^{i j}$, as the analog of the Poincaré lemma stating that if a form is closed then it is locally exact.

Note that the $y^{\lambda}$ have more information than that contained in the $g_{i j}$ and the $\pi^{i j}$ because, when the latter are given, one needs to specify in addition the location of the surface at infinity in order to determine $y^{\lambda}$ everywhere. This means that in the G-brane formulation "the problem of time" namely the reconstruction of the proper time separation between two surfaces from the knowledge of their intrinsic and extrinsic geometry is automatically solved [22]. See also [23].

After solving the constraints, only the $\pi^{i j} \dot{g}_{i j}$ term is left in the bulk Hamiltonian action. Integrating by parts in space and using (36) one obtains the action

$$
I=-\frac{1}{\kappa} \int d t d x\left(G^{i j k l} K_{i j} K_{k l} n_{\lambda} \dot{y}^{\lambda}\right),
$$

which, on account of (35) may be rewritten as

$$
I=-\frac{1}{\kappa} \int d t d x\left(g^{1 / 2}(R-2 \Lambda) n_{\lambda} \dot{y}^{\lambda}\right) .
$$

Equations (37) and (38) are alternative forms of the Gbrane action (21) because now the background is "on shell," and therefore,

$$
G^{i j k l} K_{i j} K_{k l}=g^{1 / 2}(R-2 \Lambda) .
$$

Thus we have reformulated $(2+1)$ gravity as the theory of a $(2+1)$ G-brane. 


\section{B. The $(2+1)$ black hole as a G-brane}

To gain further insight into how the G-brane formulation captures key features of the theory, we examine in its context the $(2+1)$ black hole [1, 2]. Following [24] we take for the background metric the anti-de Sitter in the form

$$
d s_{A d S}^{2}=\frac{\ell^{2}}{\sin ^{2} \chi}\left[d \chi^{2}+d \Phi^{2}-\cos ^{2} \chi d \Theta^{2}\right] .
$$

The surface variables will then be $y^{\lambda}=(\Theta, \Phi, \chi)$. Setting

$$
\begin{aligned}
\Theta & =\frac{r_{+}}{\ell^{2}} t+\frac{r_{-}}{\ell} \phi=\Theta_{0}+\Theta_{-1} \phi, \\
\Phi & =\frac{r_{-}}{\ell^{2}} t+\frac{r_{+}}{\ell} \phi=\Phi_{0}+\Phi_{-1} \phi, \\
\chi & =\arcsin \left[\left(\frac{r_{+}^{2}-r_{-}^{2}}{r^{2}-r_{-}^{2}}\right)^{1 / 2}\right]
\end{aligned}
$$

yields the black hole line element,

$$
d s_{\text {black hole }}^{2}=-f^{2} d t^{2}+\frac{d r^{2}}{f^{2}}+r^{2}\left(d \phi+N^{\phi} d t\right)^{2}
$$

where

$$
\begin{aligned}
f^{2} & =\frac{\left(r^{2}-r_{+}^{2}\right)\left(r^{2}-r_{-}^{2}\right)}{r^{2} \ell^{2}}, \\
N^{\phi} & =\frac{r_{+} r_{-}}{\ell r^{2}},
\end{aligned}
$$

provided one identifies

$$
\phi \sim \phi+2 \pi
$$

The following observations are pertinent:

(i) The functions $\Theta$ and $\Phi$ are not periodic in $\phi$. They possess "minus one modes" of strength $r_{-} / \ell$ and $r_{+} / \ell$ respectively. This may be interpreted as the imprint of a monopole type source. It is quite satisfactory to see the $r_{+}$and $r_{-}$appearing as fluxes in this manner.

(ii) If one decrees that the zero mode $\Theta_{0}$ of $\Theta$ be canonically conjugate to the minus one mode of $\Phi$, and vice versa, that is,

$$
\begin{aligned}
& {\left[\Theta_{0}, r_{+}\right]=\frac{\kappa}{2 \pi},} \\
& {\left[\Phi_{0}, r_{-}\right]=\frac{\kappa}{2 \pi},}
\end{aligned}
$$

and sets,

$$
\begin{aligned}
H & =\frac{\pi}{\kappa \ell^{2}}\left(r_{+}^{2}+r_{-}^{2}\right), \\
J & =-\frac{2 \pi}{\kappa \ell} r_{+} r_{-}
\end{aligned}
$$

then one has,

$$
\begin{array}{lll}
\frac{\partial \Theta}{\partial t}=[\Theta, H], & \frac{\partial \Theta}{\partial \phi}=-[\Theta, J], \\
\frac{\partial \Phi}{\partial t}=[\Phi, H], & \frac{\partial \Phi}{\partial \phi}=-[\Phi, J] .
\end{array}
$$

This is again satisfactory since $H$ and $J$ are precisely the mass and the angular momentum of the black hole. The symplectic structure (42) and (43) will be useful in the more general setting of Sec. VF below.

\section{Symmetry, degrees of freedom and dynamics at spatial infinity}

In $2+1$ dimensions with a negative cosmological constant, the gravitational field has an asymptotic symmetry at large spacelike distances whose Lie algebra is infinite dimensional [25]. This is a peculiarity of this low dimension, because in higher dimensions the corresponding symmetry would just be that of anti-de Sitter space. It was shown in 25 that, in $(2+1)$ spacetime dimensions, the boundary of an "asymptotically anti-de Sitter space" may be taken to be a flat cylinder. At large spatial distances the surface variables of the $(2+1)$ G-brane describe asymptotically a generic cut of that flat cylinder. This simple statement captures in a nutshell the symmetries of the boundary. The generic cut may be implemented, for example, by allowing the functions $\Theta$ and $\Phi$ appearing in (39) and (40) to possess all the Fourier modes higher than 1. The asymptotic frame in which the higher modes are absent and (39) and (40) hold could be called the "rest frame." One may go from the rest frame to a generic frame by means of a "boost," generated by the higher Fourier modes of the asymptotic symmetry generators.

[Incidentally, the previous remarks underline the fact that the presence of the infinite-dimensional asymptotic symmetry algebra, and of the surface field degrees of freedom that accompany it for $D=3$, does not account for the Bekenstein-Hawking entropy any more than the states of different linear momentum does for $D \geq 4$, in which case the asymptotic symmetry algebra is finite dimensional (see [26] for $\Lambda=0$, and [27] for $\Lambda<0$ ). One would rather expect the leading contribution to the entropy to come, in a way yet to be properly understood, from the zero mode (see [28] in this context)].

In order to be realized canonically, the infinite dimensional symmetry algebra requires an infinite number of surface degrees of freedom, which are present although the gravitational field does not have bulk degrees of freedom. These degrees of freedom emerge naturally by recalling that the condition for flatness of the cylinder at infinity is precisely the equation of motion of the $(1+1)$ gravity theory discussed in the previous section, for $\Lambda=0$, that is,

$$
{ }^{(2)} R=0 \text {. }
$$

Therefore the boundary degrees of freedom are contained in the field $\varphi$ appearing in (25).

If one passes from the Lagrangian density (28) to the Hamiltonian one finds

$$
H=\int d x\left(\eta^{\perp} t_{\perp}+\eta^{1} t_{1}\right)
$$


with

$$
\begin{aligned}
t_{\perp} & =2 k \pi^{2}+\frac{1}{8 k} \varphi^{\prime 2}-\frac{1}{2 k} \varphi^{\prime \prime}, \\
t_{1} & =\pi \varphi^{\prime}-2 \pi^{\prime},
\end{aligned}
$$

which obey the same algebra (30)-(32). In the Hamiltonian formulation these generators are the ancestors of the $(1+1)$ G-brane generators appearing in (30)-(32). Actually, of course, the reasoning is the other way around: the algebra of the G-brane generators $s_{\perp}, s_{1}$ is inherited from that of its ascendants $t_{\perp}, t_{1}$.

\section{Surface integrals as symmetry generators and their relation with the $(1+1) \mathrm{G}$-brane}

In order to account for the most general permissible motion at infinity, one must add to the bulk Hamiltonian of gravity a surface term at infinity [26]. The surface term for $(2+1)$ gravity was given in [25] and takes the form

$$
H=\int_{\infty} d \phi\left(\eta^{\perp} \mathcal{H}_{\perp \infty}+\eta^{1} \mathcal{H}_{1 \infty}\right) .
$$

In general, the surface term, which is the generator of asymptotic motions, may be realized canonically only if one fixes the coordinate system in the bulk relative to the one at infinity, and employs the Dirac bracket. It was found in [25] that $\mathcal{H}_{\perp \infty}, \mathcal{H}_{1 \infty}$ obey the algebra (30)-(32) with a central charge,

$$
k=\frac{\kappa}{\ell} .
$$

In the present context, 49 may be thought of as determining the $(1+1)$ gravitational constant $k$ in terms of its $(2+1)$ counterpart $\kappa$.

One may realize canonically the generators of asymptotic motions precisely by means of the generators $t_{\perp}, t_{1}$, by simply defining the variables $\varphi$ and $\pi$ in terms of the asymptotic parts of $\pi^{i j}$ and $g_{i j}$ appearing in $\mathcal{H}_{\perp \infty}$ and $\mathcal{H}_{1 \infty}$ through

$$
\begin{aligned}
t_{\perp} & =\mathcal{H}_{\perp \infty}, \\
t_{1} & =\mathcal{H}_{1 \infty},
\end{aligned}
$$

regarded as differential equations for $\varphi$ and $\pi$ with $\mathcal{H}_{\perp \infty}$ and $\mathcal{H}_{1 \infty}$ given.

The realization (50) may be considered as "intrinsic," or "gauge invariant," in the sense that no reference is made in it of the boundary conditions used in approaching the boundary, or of the way in which the "bulk slicing" is fixed in order to calculate a Dirac bracket. The fact that the cylinder at infinity is the boundary of the bulk is imprinted through Eq. (49).

\section{E. Treatment of zero modes}

To implement (50) it is useful to treat the zero modes separately. That is we will assume that both $\varphi$ and $\pi$ do not possess a zero mode when expanded in a Fourier series, and reinstate afterwards the zero modes by replacing

$$
\begin{aligned}
\varphi^{\prime} & \rightarrow \varphi^{\prime}-\frac{2}{\ell} r_{+}, \\
\pi & \rightarrow \pi+\frac{1}{2 k \ell} r_{-}
\end{aligned}
$$

in the Hamiltonian generators (46) and (47). Then in (50) there are as many equations as unknowns. The anti-de Sitter radius $\ell$ is brought into the above equations because we have replaced the spatial coordinate $x$ appearing in (45) by an angle $\phi$ by setting $x=\ell \phi$.

The complete action takes then the form

$$
I=\int d t\left(\frac{2 \pi}{\kappa} r_{+} \dot{\Theta}_{0}+\frac{2 \pi}{\kappa} r_{-} \dot{\Phi}_{0}+\int d \phi \pi \dot{\varphi}-H\right)
$$

where $H$ is given by (48) with the replacements (51) and (52) implemented. When $\varphi$ and $\pi$ are set equal to zero the dynamics derived from (53) is just the one described in Sec. VB.

In the treatment just presented, because the boundary is flat, one is naturally led by (44) to a description in terms of a free field $\varphi$ which has a direct metric interpretation [36]. This field may be thought of as the aggregate of the two chiral bosons emerging at the end of the analysis of the asymptotic structure of the Chern-Simons formulation given in [29] where, incidentally, accounting for the "holonomies" $r_{+}, r_{-}$remained a bit of an issue. See also [30] and references therein.

Within the Chern-Simons framework, a different proposal for the dynamics of the boundary has also been arrived at [31]. It is the Liouville theory, in which a term proportional to $e^{\varphi}$ is included in the Hamiltonian generator (46). In the present context this would correspond to replacing (44) by

$$
{ }^{(2)} R=\text { constant. }
$$

Although we have not explored this possibility, it is tempting to speculate that such a term could arise by replacing the flat cylinder at infinity by one of constant curvature, through a different choice of boundary conditions. This would be in line with the fact that one can canonically relate the Liouville theory with the one corresponding to ${ }^{(2)} R=0$ by a Bäcklund transformation (see e.g [20] and references therein).

\section{F. Coupling of the boundary degrees of freedom to a boundary G-brane}

The description of the boundary dynamics and the asymptotic symmetries in terms of a $(2+1)$ G-brane becomes sharper if one introduces a $(1+1)$ G-brane on the 
boundary. The key point is the observation that the combinations,

$$
\begin{aligned}
h_{\perp} & =\left.s_{\perp}\right|_{k \rightarrow-k}+t_{\perp}, \\
h_{1} & =s_{1}+t_{1},
\end{aligned}
$$

close according to the algebra (30)-(32) without central charge. Here the notation $k \rightarrow-k$ means that one replaces in $s_{\perp}$ the constant $k$ by its negative. This replacement maintains the surface deformation algebra, but changes the sign of the central charge.

Since the algebra of the generators $h_{\perp}, h_{1}$ has no central charge, it is consistent to demand that these $h$ 's be constrained to vanish,

$$
\begin{aligned}
h_{\perp} & \approx 0, \\
h_{1} & \approx 0 .
\end{aligned}
$$

Thus the canonically conjugate field $\varphi$ and $\pi$ out of which the generators $t_{\perp}$ and $t_{1}$ are constructed may be regarded as matter fields defined on the $(1+1)$ G-brane and accompanying it through its deformations.

In terms of the above generators, the action for $(2+1)$ gravity becomes the sum of three terms, integrated respectively over manifolds of dimension three (the bulk), two (the boundary), and unity (the history of the zero modes):

$$
\begin{aligned}
I= & \int d t\left[\int d^{2} x w_{\lambda} \dot{y}^{\lambda}+\int d^{1} x\left(w_{a} \dot{y}^{a}+\pi \dot{\varphi}\right)\right. \\
& \left.+\frac{2 \pi}{\kappa} r_{+} \dot{\Theta}+\frac{2 \pi}{\kappa} r_{-} \dot{\Phi}-H\right],
\end{aligned}
$$

with

$$
\begin{aligned}
H= & \int d^{2} x\left(N^{\perp} h_{\perp}^{(2+1)}+N^{i} h_{i}^{(2+1)}\right) \\
& +\int d x\left(\eta^{\perp} h_{\perp}^{(1+1)}+\eta^{1} h_{1}^{(1+1)}\right),
\end{aligned}
$$

and

$$
\begin{aligned}
\eta^{\perp} & =g^{-1 / 2} N^{\perp}(\infty), \\
\eta^{1} & =N^{1}(\infty) .
\end{aligned}
$$

In (58) we have allowed for a generic background coordinate system $y^{a},(a=0,1)$, with corresponding metric $\gamma_{a b}$, on the flat boundary at infinity. If the boundary were described as $y^{\lambda}=y^{\lambda}\left(t, \phi, r_{0}\right)$ with $r_{0} \rightarrow \infty$, then the $y^{a}$ would be related to $t$ and $\phi$ by a coordinate transformation, which might depend on $r_{0}$ as a parameter.

The formulation of $(2+1)$ gravity in terms of $(2+1)$ and $(1+1)$ G-branes summarized in the action (58) is "fully parametrized" in the sense of possessing a Hamiltonian that vanishes weakly, even when the boundary dynamics is included [the boundary action is invariant under spacetime reparametrizations because due to the change $k \rightarrow-k$ in (54), the terms of the form (29) coming from the brane and the matter contributions mutually cancel].
Such a description would not have been possible if one had attempted to achieve it through a Dirac or a Nambu brane, because then the central charge would not drop out. In other words, the "background energy" of the $(1+1)$ G-brane is essential. Note that the background energy which actually appears in this construction is $-\mathcal{L}$, the negative of the standard one, because the sign of $k$ in $s_{\perp}$ has been changed. There is no harm in this because $\mathcal{L}$ does not have a definite sign to begin with.

\section{CONCLUDING REMARKS}

We have introduced, for any spacetime dimension $D$, the concept of a G-brane, which is a space-filling brane whose action is a direct descendant of the action for the gravitational field. The G-brane may be thought of as the remanent of the gravitational field when the propagating gravitons are removed. We were led to the G-brane in the midst of an effort to gain understanding of the puzzling feature, evidenced by the existence of a black hole in $2+1$ spacetime dimensions [1, 2], that key aspects of gravitation are retained even when there are no propagating gravitational field degrees of freedom. The G-brane concept was applied for the lower dimensions $D=3$ and $D=2$, where the following results were obtained: (i) For $D=3$ the G-brane yields a reformulation of gravitation theory in which the Hamiltonian constraints can be solved explicitly, while keeping the spacetime structure manifest. (ii) For $D=2$ one finds a new realization of the conformal algebra, i.e. a conformal field theory, in terms of two scalar fields and their conjugates, which possesses a classical central charge. (iii) In the G-brane reformulation of $(2+1)$ gravity, the boundary degrees of freedom of the gravitational field in asymptotically antide Sitter space appear as matter coupled to the $(1+1)$ G-brane on the boundary.

Finally, a word about future developments. As it was just indicated, although the concept and general properties of the G-brane were presented, no applications were given for the higher dimensions $D \geq 4$. Therefore a problem that brings itself immediately for analysis is how, for $D \geq 4$, the G-brane inherits from the gravitational field two key aspects, namely, the entropy and the asymptotic structure at spacelike infinity. Additionally one would like to develop the notion of a supersymmetric G-brane for all $D \geq 2$. Finally a less well-defined question is to ask if and how, the idea of the G-brane as "gravitation without gravitons" relates to two recent different lines of inquiry, namely: (i) the role of "soft gravitons" and their relation with asymptotic symmetries discussed in [32] and [33] and, (ii) the interplay of "longitudinal gravitons" with black hole entropy discussed in [34] and [35]. We hope to address these issues in the near future. 


\section{Acknowledgments}

The Centro de Estudios Científicos (CECs) is funded by the Chilean Government through the Centers of Ex- cellence Base Financing Program of Conicyt. C.B. wishes to thank the Alexander von Humboldt Foundation for a Humboldt Research Award. The work of A.P. is partially funded by the Fondecyt Grant № 11130262 .
[1] M. Banados, C. Teitelboim and J. Zanelli, "The Black hole in three-dimensional spacetime," Phys. Rev. Lett. 69, 1849 (1992) hep-th/9204099.

[2] M. Banados, M. Henneaux, C. Teitelboim and J. Zanelli, "Geometry of the $(2+1)$ black hole," Phys. Rev. D 48, 1506 (1993) [Phys. Rev. D 88, no. 6, 069902 (2013)] gr-qc/9302012.

[3] T. Regge and C. Teitelboim, "General Relativity à la String: A Progress Report," In *Trieste 1975, Proceedings, Marcel Grossmann Meeting On General Relativity, edited by Remo Ruffini, North-Holland (Amsterdam 1977), pp 77-87.

[4] S. Deser, F. A. E. Pirani and D. C. Robinson, "New embedding model of general relativity," Phys. Rev. D 14, 3301 (1976).

[5] S. A. Paston and A. N. Semenova, "Constraint algebra for Regge-Teitelboim formulation of gravity," Int. J. Theor. Phys. 49, 2648 (2010) doi:10.1007/s10773-0100456-5 arXiv:1003.0172 [gr-qc]l.

[6] S. A. Paston and E. N. Semenova, "External time canonical formalism for gravity in terms of embedding theory," Gravit. Cosmol. 21, no. 3, 181 (2015) doi:10.1134/S020228931503007X arXiv:1509.01529 [gr$\mathrm{qc}]]$.

[7] P. A. M. Dirac, "The Hamiltonian form of field dynamics," Can. J. Math. 3, 1 (1951).

[8] P. A. M. Dirac, "Generalized Hamiltonian dynamics," Can. J. Math. 2, 129 (1950).

[9] C. Teitelboim, "How commutators of constraints reflect the space-time structure," Annals Phys. 79, 542 (1973).

[10] C. Teitelboim, "The Hamiltonian Structure Of Spacetime," Ph. D. Thesis, Princeton University (unpublished).

[11] J. Schwinger, "Commutation Relations and Conservation Laws," Phys. Rev. 130, 406 (1963).

[12] D. Lovelock, "The Einstein tensor and its generalizations," J. Math. Phys. 12, 498 (1971).

[13] C. Teitelboim and J. Zanelli, "Dimensionally continued topological gravitation theory in Hamiltonian form," Class. Quant. Grav. 4, L125 (1987).

[14] P. A. M. Dirac, "A reformulation of the Born-Infeld electrodynamics," Proceedings of the Royal Society of London A: Mathematical, Physical and Engineering Sciences. Vol. 257. No. 1288. The Royal Society, 1960.

[15] J. D. Brown and C. Teitelboim, "Dynamical Neutralization of the Cosmological Constant," Phys. Lett. B 195, 177 (1987).

[16] J. D. Brown and C. Teitelboim, "Neutralization of the Cosmological Constant by Membrane Creation," Nucl. Phys. B 297, 787 (1988).

[17] A. Gomberoff, M. Henneaux, C. Teitelboim and F. Wilczek, "Thermal decay of the cosmological constant into black holes," Phys. Rev. D 69, 083520 (2004) hep-th/0311011.

[18] A. Einstein, "Does the inertia of a body depend upon its energy-content?," Annalen der Physik 18, 13, 639-641, (1905).

[19] C. Teitelboim, "The Hamiltonian Structure Of Twodimensional Space-time And Its Relation With The Conformal Anomaly," In *Christensen, S.M. ( Ed.): Quantum Theory Of Gravity*, Adam Hilger Ltd, Bristol, 327344.

[20] R. Jackiw, "Liouville Field Theory: A Two-dimensional Model For Gravity?," In *Christensen, S.M. ( Ed.): Quantum Theory Of Gravity*, Adam Hilger Ltd, Bristol, 403-420.

[21] C. Bunster and A. Pérez, "Conformal field theory of a space-filling string of gravitational ancestry," in Proceedings of the Fourteenth Marcel Grossman Meeting on General Relativity, edited by Massimo Bianchi, Robert T. Jantzen, Remo Ruffini (World Scientific, Singapore, 2016).

[22] R. F. Baierlein, D. H. Sharp and J. A. Wheeler, "ThreeDimensional Geometry as Carrier of Information about Time," Phys. Rev. 126, 1864 (1962).

[23] C. W. Misner, K. S. Thorne and J. A. Wheeler, "Gravitation," San Francisco 1973, 1279p

[24] S. Carlip and C. Teitelboim, "Aspects of black hole quantum mechanics and thermodynamics in (2+1)-dimensions," Phys. Rev. D 51, 622 (1995) gr-qc/9405070.

[25] J. D. Brown and M. Henneaux, "Central Charges in the Canonical Realization of Asymptotic Symmetries: An Example from Three-Dimensional Gravity," Commun. Math. Phys. 104, 207 (1986).

[26] T. Regge and C. Teitelboim, "Role of Surface Integrals in the Hamiltonian Formulation of General Relativity," Annals Phys. 88, 286 (1974).

[27] M. Henneaux and C. Teitelboim, "Asymptotically antiDe Sitter Spaces," Commun. Math. Phys. 98, 391 (1985).

[28] C. Teitelboim, "Statistical thermodynamics of a black hole in terms of surface fields," Phys. Rev. D 53, 2870 (1996) hep-th/9510180.

[29] M. Henneaux, L. Maoz and A. Schwimmer, "Asymptotic dynamics and asymptotic symmetries of threedimensional extended AdS supergravity," Annals Phys. 282, 31 (2000) hep-th/9910013.

[30] S. Carlip, "Conformal field theory, $(2+1)$-dimensional gravity, and the BTZ black hole," Class. Quant. Grav. 22, R85 (2005) gr-qc/0503022.

[31] O. Coussaert, M. Henneaux and P. van Driel, "The Asymptotic dynamics of three-dimensional Einstein gravity with a negative cosmological constant," Class. Quant. Grav. 12, 2961 (1995) gr-qc/9506019.

[32] A. Strominger, "On BMS Invariance of Gravitational Scattering," JHEP 1407, 152 (2014) doi:10.1007/JHEP07(2014)152 arXiv:1312.2229 [hepth]].

[33] T. He, V. Lysov, P. Mitra and A. Strominger, " BMS supertranslations and Weinberg's soft graviton theorem," 
JHEP 1505, 151 (2015) doi:10.1007/JHEP05(2015)151 arXiv:1401.7026 [hep-th]].

[34] G. Dvali and C. Gomez, "Black Hole's Quantum N-Portrait," Fortsch. Phys. 61, 742 (2013) doi:10.1002/prop.201300001 arXiv:1112.3359 [hep-th]].

[35] G. Dvali and C. Gomez, "Quantum Compositeness of Gravity: Black Holes, AdS and Inflation," JCAP 1401, 023 (2014) doi:10.1088/1475-7516/2014/01/023 arXiv:1312.4795 [hep-th]].

[36] According to (25) the field is the logarithm of the metric component of a cut of the boundary. To reach that cut one would have to tune the $y^{\lambda}$ coordinate system so that, as the boundary is approached, it matches the "intrinsic frame" selected by (50). For example, the coordinates which realize the boundary conditions of 25] will not do. That a coordinate system that does the job indeed exists is guaranteed by the fact that the dynamics of $\varphi$ ensures that the boundary is flat. 